Journal of Quantitative Spectroscopy and Radiative Transfer 109, 2008, 2731-2742

ISSN: 0022-4073

doi:10.1016/j.jqsrt.2008.07.007

(c) 2008 Elseiver Ltd. All rights reserved.

http://www.elsevier.com/wps/find/homepage.cws home

www.elsevier.com/locate/jqsrt

\title{
Electron-ion recombination and photoionization of Fe XXI
}

\author{
Sultana N. Nahar \\ Department of Astronomy, The Ohio State University, Columbus, OH 43210, USA
}

\begin{abstract}
Results for electron-ion recombination and photoionization of (Fe XXI + hv↔ Fe XXII + e), with emphasis in hightemperature region, are presented from ab initio unified method. The unified method, based on close coupling (CC) approximation and R-matrix method, (i) subsumes both the radiative recombination (RR) and dielectronic recombination (DR), (ii) enables self-consistent sets of photoionization and recombination cross sections from using an identical wavefunction for both the processes, and (iii) provides state-specific recombination rates of a large number of bound states. A large CC wavefunction expansion, which includes the ground and 28 core excitations of $n=2$ and 3 complexes and span a wide energy range, has been used. Compared to $\Delta n=2-2, \Delta n=2-3$ core excitations are found to introduce strong resonant structures and enhance the background photoionization cross sections $\left(\sigma_{\mathrm{PI}}\right)$ in the high-energy region. These features along with prominent photoexcitation-of-core (PEC) resonances at $\mathrm{n}=3$ core thresholds have increased the unified total recombination rate coefficients $\alpha_{\mathrm{R}}(T)$ ) at temperatures $T>10^{6} \mathrm{~K}$, region of maximum abundance of the ion in collisional equilibrium, by a factor of 1.6 over previous calculations. State-specific recombination rate coefficients $\alpha_{R}(n L S)$, which include both the RR and DR, are presented for the first time for 685 bound states with $n \leq 10$ and $l \leq 9$. The unified total recombination rate with photoelectron energy $\alpha_{R}(E)$ is presented and the role of low-energy near-threshold fine structure resonances is illustrated. The present results should provide a reasonably complete self-consistent set of recombination rates and photoionization cross sections for astrophysical modelings of high-temperature plasmas from optical to farultraviolet wavelength regions.
\end{abstract}

\section{Introduction}

Carbon-like iron ion, Fe XXI, is astrophysically abundant in variety of high-temperature sources such as sun, nebulae, active galactic nuclei, stars, etc. Fe XXI lines in solar flares are used in determination of electron densities (e.g. [1]). It may also exist in low temperature photoionized plasmas. Soft X-ray line 12.282 $\AA$ (3d-2p) of Fe XXI has been detected in the photoionized plasmas of a number of nearby obscured active galactic nuclei by XMM-Newton [2]. Modeling of astrophysical spectra requires total $(\mathrm{e}+\mathrm{ion})$ recombination rates, incorporating both the radiative recombination (RR) and dielectric recombination (DR), to calculate quantities such as ionization fractions of elements $[3,4]$. The aim of the present work is to provide selfconsistent results for photoionization and electron-ion recombination (e.g. [5]) for accurate astrophysical applications.

In contrast to the unified method of (e + ion) recombination [6-9] the available rates are for RR and DR calculated separately. The total rates are then approximated by summing the nonresonant $\mathrm{RR}$ and the resonant $\mathrm{DR}$ rate coefficients, often supplemented by a very low temperature DR component. The earlier works on Fe XXI were carried out for RR rates obtained using central-field and/or hydrogenic approximations, for example, by Arnaud and Raymond [3], Gu [10], Badnell [11], and for DR rates obtained using isolated resonance approximation (IRA), 
for example, by Jacobs et al. [12], Badnell [13], Altun et al. [14], Gu [15]. A recent measurement for Fe XXI recombination was carried out in the low energy region by Savin et al. [16] using the facility at the Test Storage Ring (TST) in Heidelberg. They provide the fit for DR rate coefficient, $\alpha_{D R}(T)$, for $n=2$ excitations.

The present calculation employs a wavefunction expansion that includes core excitations of both $n=2$ and 3 states spanning over an energy range of about $83 \mathrm{Ry}$. The details of the total photoionization cross sections of a large number of bound states, 835 in total, for Fe XXI have been reported earlier by Nahar [17] while present report provides results of electron-ion recombination and partial photoionization cross sections needed for recombination. Unified method was used earlier for total and state-specific recombination rate coefficients for other carbon-like ions, from C I to S XI [18,19], Ar XIII [20].

\section{Theory}

The computational framework of unified method for total electron-ion recombination is based on the close coupling (CC) approximation and R-matrix method as implemented in the Opacity Project (OP) [21] and the Iron Project (IP) [22]. A brief outline of the theory relevant to the present calculations is given below.

In the CC approximation, the 'target' or the 'core' ion is represented by an N-electron system interacting with the $(N+1)$ th electron. The $(N+1)$ th electron may be bound or in the continuum depending on its negative or positive energy $(E)$. The total wavefunction, $\Psi_{E}$, of the $(N+1)$ electrons system in a symmetry $S L \pi$ is represented by an expansion as

$$
\Psi_{E}(\mathrm{e}+\mathrm{ion})=A \sum_{i} \chi_{i}(\operatorname{ion}) \theta_{i}+\sum_{j} c_{j} \Phi_{j},
$$

where the target ion eigenfunction, $\chi i$, is coupled with the $(N+1)$ th electron function, $\theta_{i}$. The sum is over the ground and excited core states. The $(N+1)$ th electron with kinetic energy $k_{\mathrm{i}}^{2}$ is in a channel labeled as $S_{i} L_{i} \pi_{i} k_{i}^{2} \ell_{i}(S L \pi)$. In the second sum, the $\Phi j$ 's are bound channel functions of the $(N+1)$-electrons system that account for short range correlation and the orthogonality between the continuum and the bound electron orbitals.

The relativistic Hamiltonian in the Breit-Pauli R-matrix (BPRM) method under the IP is given by

$$
H_{N+1}^{\mathrm{BP}}=\sum_{i=1}^{N+1}\left\{-\nabla_{i}^{2}-\frac{2 Z}{r_{i}}+\sum_{j>1}^{N+1} \frac{2}{r_{i j}}\right\}+H_{N+1}^{\text {mass }}+H_{N+1}^{\text {Dar }}+H_{N+1}^{50} .
$$

where the additional one-body terms

$$
H_{N+1}^{\text {mass }}=-\frac{\alpha^{2}}{4} \sum_{i} p_{i}^{4}, \quad H_{\text {Dar }}=-\frac{\alpha^{2}}{4} \sum_{i} \nabla^{2}\left(\frac{Z}{r_{i}}\right), \quad H_{s o}=\alpha^{2} \sum_{i=1} \frac{Z}{r_{i}^{3}} l(i) \cdot s(i)
$$

are the relativistic mass correction, Darwin, and spin-orbit interaction terms, respectively. The present calculations are carried out in LS coupling, but including mass correction and Darwin terms. Due to strong fine structure couplings in low energy, relativistic calculations with the three correction terms were carried out only for $2 s 2 p^{35} s^{0}$ state. 
Substitution of $\Psi_{E}(\mathrm{e}+$ ion $)$ expansion in the Schrodinger equation

$$
H_{N+1} \Psi_{E}=E \Psi_{E}
$$

results a set of coupled equations that are solved using the $R$-matrix method. The solution is a continuum wavefunction, $\Psi_{F}$, for an electron at positive energies $(E>0)$, or a bound state, $\Psi_{B}$, at a negative total energy $(E \leq 0)$. The complex resonant structures in photoionization and recombination result from channel couplings between continuum channels that are open $\left(k_{\mathrm{i}}^{2}>0\right)$, and ones that are closed $\left(k_{\mathrm{i}}^{2}<0\right)$, at electron energies $k_{\mathrm{i}}^{2}$ corresponding to the Rydberg series converging on to the target thresholds.

Transition matrix elements for photoionization or electron-ion recombination can be obtained using the bound and continuum wavefunctions as $\left(\Psi_{B}\|\mathbf{D}\| \Psi_{F}\right)$ where $\mathbf{D}=\sum_{i} r_{i}$ is the dipole operator, and the sum represents the number of electrons. The transition matrix element can be reduced to generalized line strength as

$$
S=\left|\left\langle\Psi_{j}\left\|\mathbf{D}_{i}\right\| \Psi_{i}\right\rangle\right|^{2}=\left|\left\langle\Psi_{\mathrm{f}}\left|\sum_{j=1}^{N+1} \mathrm{r}_{j}\right| \Psi_{i}\right\rangle\right|^{2}
$$

where $\Psi_{i}$ and $\Psi_{f}$ are the initial and final state wavefunctions. Photoionization cross section ( $\left.\sigma_{\mathrm{PI}}\right)$ is proportional to the generalized line strength as

$$
\sigma_{\mathrm{Pl}}=\frac{4 \pi^{2}}{3 c} \frac{1}{g_{i}} \omega \mathbf{S}
$$

where $g_{i}$ is the statistical weight factor of the bound state and $\omega$ is the incident photon energy.

Recombination cross sections, $\sigma_{\mathrm{RC}}$, can be obtained from detailed partial photoionization cross section using the Milne relation,

$$
\sigma_{\mathrm{RC}}=\sigma_{\mathrm{PI}} \frac{g_{i}}{g_{j}} \frac{h^{2} \omega^{2}}{4 \pi^{2} m^{2} c^{2} v^{2}}
$$

where $g_{i}$ is the statistical weight factor of the recombined state and $v$ is the photoelectron velocity. The recombining ion is assumed to be in the ground state. The detailed autoionizing structures in photoionization cross sections are integrated to recombination cross sections through the Milne relations. Hence the sum corresponds to inclusion of both the RR and DR in an unified and ab initio manner.

The state-specific recombination rate coefficient of state $i$ is obtained by averaging the recombination cross sections over the Maxwellian electron distribution, $f(v)=(4 /$ $\sqrt{ } \pi)(m / 2 k T)^{3 / 2} v^{2} e^{-m v 2 / 2 k T}$, as

$$
\alpha_{\mathrm{R}}(i ; T)=\int_{0}^{\infty} v f(v) \sigma_{\mathrm{RC}}(i) \mathrm{d} v .
$$

The total recombination rate can be obtained from sum of these individual rates, that is, $\alpha_{R}(T)=$ 
$\Sigma_{\mathrm{i}} \alpha_{\mathrm{R}}(i, T)$ where the sum is over infinite number of recombined states.

The unified method divides the infinite recombined states into two groups: group (A) states with $n \leq n_{0}$, and group (B) states with $n_{0}<n \leq \infty$, with $\mathrm{n}_{0} \sim 10$. The recombination rate coefficients of group (A) states are obtained from the photoionization cross sections as described above.

Recombination into group (B) states, which are in a small energy range, is dominated by DR via high-n resonances belonging to various core thresholds while the background recombination, that is, RR is negligibly small. The precise DR theory by Bell and Seaton [23], based on quantum defect theory and CC approximation, was extended by Nahar and Pradhan [7] for DR collision strengths, $\Omega(D R)$, as

$$
\Omega(D R)=\sum_{S L \pi} \sum_{n} \frac{1}{2}(2 S+1)(2 L+1) P_{n}^{S L R}(D R),
$$

where $P_{n}^{S I \pi}(D R)=\left(1-\mathscr{S}_{\mathrm{ee}}^{4} \mathscr{S}_{\mathrm{ee}}\right)_{n}$ is the DR probability in entrance channel n. $\mathscr{S}_{\mathrm{ee}}$ is the electron scattering matrix including radiation damping. Calculations for $\Omega(D \mathrm{R})$ are carried out in the CC approximation using the same wavefunction expansion used for photoionization cross sections. The recombination cross section is related to $\Omega(D \mathrm{R})$ as

$$
\sigma_{\mathrm{RC}}(D R)=\frac{\pi}{\mathrm{g}_{i} k^{2}} \Omega(D R) \mathrm{a}_{0}^{2},
$$

$\Omega(D R)$ is summed over all contributing symmetries $S L \pi$. The total DR contributions of $n>10$ states is usually much smaller than that for $n<10$ states except at the DR peak where it can still be smaller but of the same order.

The RR-type 'background' contributions from the high- $n$ group (B) states, $n_{0}<n \leq \infty$, to the total recombination rate are included in hydrogenic approximation [24]. These contributions are usually negligible except at very low temperatures where electron energies are not high enough for core excitations.

The unified total recombination rate coefficients with photoelectron energy, $\alpha_{R}(E)$, can be obtained as

$$
\alpha_{\mathrm{R}}(E)=v \sigma_{\mathrm{RC}}(E),
$$

where $v$ is the photoelectron energy. $\alpha_{\mathrm{R}}(E)$, being proportional directly to $\sigma_{\mathrm{RC}}(E)$, reveals detailed resonant features in electron-ion recombination spectrum and is of considerable interest as it is a measurable quantity at facilities such as Test Storage Ring at Heidelberg.

\section{Computations}

The computations for photoionization and electron-ion recombination are carried out through a number of stages starting with determination of target wavefunction. The wavefunction for target Fe XXII was obtained from atomic structure calculations using code SUPERSTRUCTURE [25]. It includes the ground and 28 excited states of Fe XXII from $n=2$ and 3 complexes as given in Table 1 . The table and computation of photoionization cross sections have been explained in Nahar [17]. 
State-specific recombination rate coefficients for all bound states with $n \leq 10$ are obtained from code RECOMB [24] and are added together for the total. The contributions of high- $n$ states below the target thresholds are obtained from the resonance averaged DR collision strength $\Omega(D R)$ using code STGFDR [7]. Values of $\Omega(D R)$ were calculated using the radiative decay rates (A-values) for the dipole allowed transitions to the core ground state $2 s^{2} 2 p\left({ }^{2} P^{0}\right.$ ). These $A$-values, given in

\section{Table 1}

Terms and energies of Fe XXII in the eigenfunction expansion of Fe XXI

\begin{tabular}{|c|c|c|}
\hline & Term & E (Ry) \\
\hline \multicolumn{3}{|c|}{$n-2$ states } \\
\hline 1 & $2 s^{2} 2 p\left(^{2} p^{0}{ }_{1,2}\right)$ & 0.0 \\
\hline 2 & $2 s 2 p^{2}\left(P^{\prime}\right)$ & 4.350891 \\
\hline 3 & $2 \varsigma 2 p^{2}\left(^{2} D^{\prime}\right)$ & 6.837967 \\
\hline 4 & $252 p^{2}\left(^{2} s^{5}\right)$ & 8.620770 \\
\hline 5 & $2 s 2 p^{2}\left(^{2} P^{r}\right)$ & 8.914198 \\
\hline 6 & $2 p^{3}\left(s^{4} s^{0}\right)$ & 11.442782 \\
\hline 7 & $2 p^{3}\left(^{2} D^{0}\right)$ & 12.891660 \\
\hline 8 & $2 p^{3}\left({ }^{2} P^{0}\right)$ & 14.6564263 \\
\hline \multicolumn{3}{|c|}{$n-3$ states } \\
\hline 9 & $\left.2 s^{2} 3 s s^{2} S\right)$ & $73.24894^{*}$ \\
\hline 10 & $2 s^{2} 3 p\left({ }^{2} P^{0}\right)$ & $74.83334^{*}$ \\
\hline 11 & $\left.2 . s 2 p 3 s s^{4} p^{0}\right)$ & $76.37739^{*}$ \\
\hline 12 & $2 s 2 p 3 s\left({ }^{2} p^{0}\right)$ & $77.20010^{*}$ \\
\hline 13 & $\left.2 s^{2} 3 d t^{2} D\right)$ & 77.48869 \\
\hline 14 & $2 s 2 p 3 p(D)$ & 77.81568 \\
\hline 15 & $2 s 2 p 3 p\left(c^{4} s\right)$ & $78.08464^{*}$ \\
\hline 16 & $\left.2 s 2 p 3 p c^{4} \beta\right)$ & $78.38718^{*}$ \\
\hline 17 & $\left.2 s 2 p 3 p c^{2} P\right)$ & 78.85499 \\
\hline 18 & $2 s 2 p 3 p\left({ }^{2} s\right)$ & $79.09630^{*}$ \\
\hline 19 & $2.52 p 3 s\left({ }^{2} p^{0}\right)$ & $79.41596^{*}$ \\
\hline 20 & $\left.2 s 2 p 3 p c^{2} D\right)$ & 80.21885 \\
\hline 21 & $2 s 2 p 3 d\left(^{2} p^{0}\right)$ & $80.53455^{*}$ \\
\hline 22 & $\left.2 \sin p 3 d d^{4} F^{0}\right)$ & 80.77472 \\
\hline 23 & $2 \leqslant 2 p 3 p\left(^{2} P\right)$ & $80.90579^{\circ}$ \\
\hline 24 & $252 p 3 p\left(^{2} D\right)$ & $80.97771^{*}$ \\
\hline 25 & $2 s 2 p 3 d\left(p^{4} p^{0}\right)$ & 81.31389 \\
\hline 26 & $2 s 2 p 3 p\left(^{2} S\right)$ & $81.39861^{*}$ \\
\hline 27 & $2 \leqslant 2 p 3 d\left({ }^{2} D^{0}\right)$ & 81.44906 \\
\hline 28 & $2 s 2 p 3 d\left(D^{A} D^{0}\right)$ & 81.48460 \\
\hline 29 & $\left.2 s 2 p 3 d d^{2} F^{0}\right)$ & 82.45406 \\
\hline
\end{tabular}

A large energy gap ( 60 Ry) can be noticed between $n=2$ and 3 states. The asterisks indicate calculated energies since the measured values are not available.

Table 2 
Radiative decay rates, $A_{i i}$, for dipole allowed transitions from various excited states to the ground state $2 s^{2} 2 p\left({ }^{2} P^{0}\right)$ of core Fe XXII

\begin{tabular}{|c|c|c|}
\hline Target state:f & $A_{\text {ff }}(\mathrm{d}, \mathrm{u})$. & $\left\langle\Omega(D R)_{f}\right\rangle$ \\
\hline \multicolumn{3}{|c|}{$\Delta n-0(2)$ 2 2 transitions } \\
\hline $2 s 2 p^{2}\left(^{2} D\right)$ & $1.12(-7)$ & 0.411 \\
\hline $252 p^{2}\left(^{2} P\right)$ & $4.79(-7)$ & 0.186 \\
\hline $2 s 2 p^{2}\left({ }^{2} s\right)$ & $8.62(-7)$ & 0.787 \\
\hline \multicolumn{3}{|c|}{$\Delta n-1(32)$ transitions } \\
\hline $\left.2 s^{2} 35 s^{2} s\right)$ & $5.53(-5)$ & 0.0052 \\
\hline $\left.2 s^{2} 3 d l^{2} D\right)$ & $4.38(-4)$ & 0.1735 \\
\hline $2 s 2 p 3 p\left({ }^{2} P\right)$ & $1.84(-4)$ & 0.015 \\
\hline $2 s 2 p 3 p\left({ }^{2} s\right)$ & $2.33(-4)$ & 0.004 \\
\hline $\left.2 s 2 p 3 p x^{2} D\right)$ & $2.32(-4)$ & 0.027 \\
\hline $2 s 2 p 3 p\left({ }^{2} P\right)$ & $6.00(-5)$ & 0.0033 \\
\hline $2 s 2 p 3 p\left({ }^{2} D\right)$ & $1.23(-5)$ & 0.0003 \\
\hline $2 s 2 p 3 p\left(^{2} 5\right)$ & $2.17(-5)$ & 0.0013 \\
\hline
\end{tabular}

$\{\Omega(D R)\}$ is the DR collision strength at these excited thresholds.

Table 2, were obtained from atomic structure code SUPERSTRUCTURE. The contributions from all $n \leq \infty$ were added for the total unified $\alpha_{\mathrm{R}}(E)$ and $\alpha_{R}(T)$ using program RECXS.

\section{Results and discussions}

The inverse processes of photoionization and electron-ion recombination of the highly charged Fe XXI are studied in detail. The aim of the large 29 CC wavefunction expansion is to study their properties in high temperature. Fe XXI is abundant in high temperature, $T \sim 10^{6-7} \mathrm{~K}$, in collisional plasmas (e.g. [3]). Although the $n=2$ and 3 core states separated by about 60 Ry (Table 1 ), the radiative decay rates ( $A$-values) for $\Delta n=3-2$ transitions are found to be much stronger than those for $\Delta n=2-2$ transitions, by up to a few orders of magnitude (Table 2) predicting considerable resonant effects from $n=3$ excitations.

The results are obtained in LS coupling. This ion has large number of bound states, a total of 835 with $n \leq 10,0 \leq l \leq 9$ [17]. Spectroscopic identification of these bound states of Fe XXI was carried out using quantum defect analysis. However, due to similar quantum defects of closely lying core states and mixing of states some uncertainties are expected for proper identification of the states.

\subsection{Photoionization cross sections}

Detail study of total photoionization cross sections $\sigma_{\mathrm{PI}}$ of a large number of bound states of Fe XXI is presented in Nahar[17]. However, recombination rate coefficients require partial photoionization cross sections $\sigma_{\mathrm{PI}}(g, n L S)$ where the core ion is left in the ground state. Important features in $\sigma_{\text {PI }}(n L S)$ that affect the recombination cross sections are illustrated below. $\sigma_{\text {PI }}(g, n L S)$ of all 685 states that couple to the core ground state are available electronically at NORAD website [27]. 


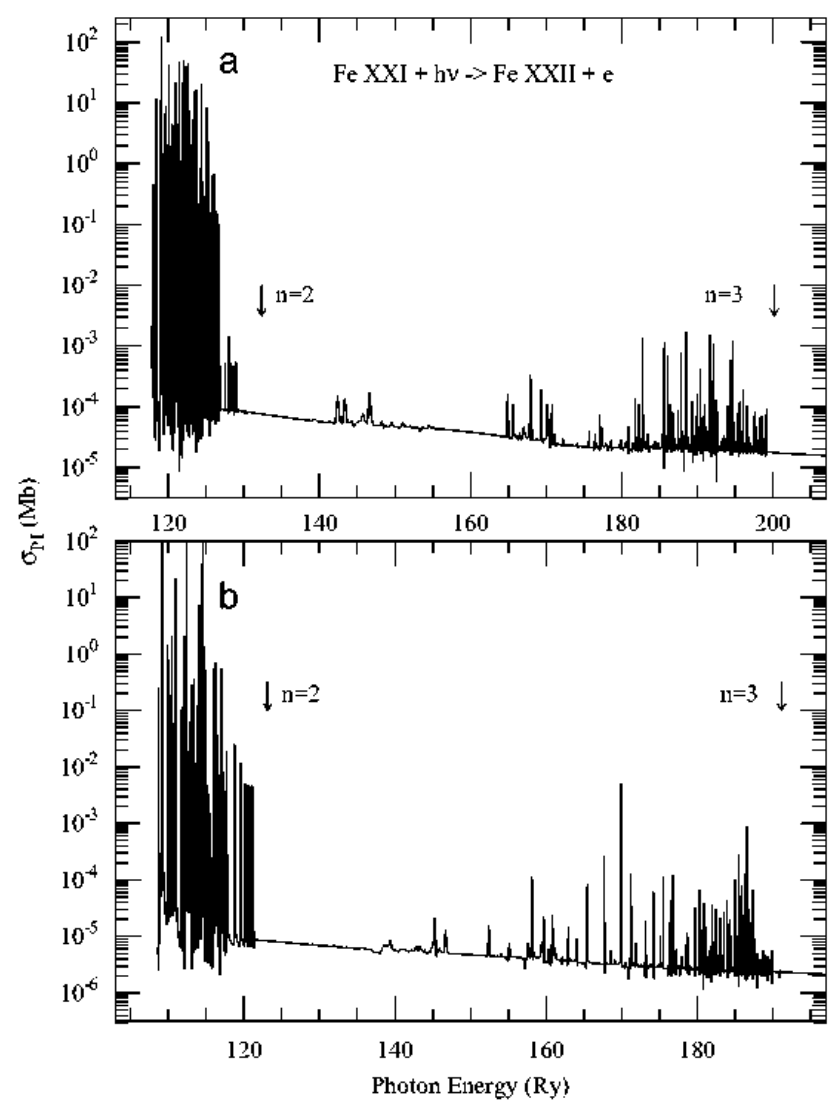

Fig. 1. Partial photoionization cross sections, $\sigma_{\mathrm{PI}}$, of the excited metastable states of Fe XXI: (a) $2 s 2 p^{3}\left({ }^{3} D^{0}\right)$, (b) $2 p^{4}\left({ }^{1} D\right)$. These states contribute considerably to recombination due to extensive low energy resonances and slow decay of the background cross sections at higher energy.

The existing $\sigma_{\text {PI }}$ available at TOPbase (vizier.u-strasbg.fr/topbase/topbase.html) under the OP [28] correspond to only total photoionization. Comparison of the present total $\sigma_{\text {PI }}$ made with those from TOPbase showed considerable improvement of accuracy and higher resolution in the present work [17]. In addition, the energy region beyond $n=2$ core states, where TOPbase data are extrapolated with hydrogenic behavior, is filled with rich resonant features due to core $n$ $=3$ complex indicating that the photoionization rates from OP cross sections will be grossly underestimated.

Metastable states play an important role in photoionization and electron-ion recombination rates because of low energy resonances and slow decay of the background cross section with energies. Fig. 1 presents partial $\sigma_{\text {PI }}$ of metastable states (a) $2 s 2 p^{3}\left({ }^{3} D^{0}\right.$ ) and (b) $2 p^{4}\left({ }^{1} D\right)$ illustrating their dominance. The low energy region of each state is filled with strong and dense resonances belonging to eight core states of $n=2$ complex and contribute considerably to recombination rates. The contributions may exceed to that of the ground state. In contrast to the ground state where $n=3$ core states do not affect the background feature [17], these metastable states show significant number the resonances belonging to the 21 core states of $n=3$ complex. These states continue to dominate the rates in to the high-temperature region.

Fig. 2 presents partial photoionization cross sections of two excited valence electron states, (a) $2 s^{2} 2 p 3 d\left({ }^{3} D^{0}\right)$ and (b) $2 s^{2} 2 p 3 d\left({ }^{3} F^{0}\right)$ of Fe XXI. Features for these states are quite different from those of equivalent electron states. Here the role of $n=3$ core states becomes 
more prominent. The arrows, for each state in the figure, point the energy limits of the $n=2$ and 3 core thresholds. The extensive resonant features due to $n=3$ states in the wide energy range are considerably more prominent than those due to $n=2$ core states. This feature was noted earlier for other ions $[7,20]$. But such prominence was not expected for highly charged Fe XXI where the core states of $n=2$ and 3 complexes are separated by about $60 \mathrm{Ry}$. The figures indicate strong correlations of various higher channels of excited $n=3$ core states for excited valence electron states. The large radiative decay rates of $n=3$ states are the reasons for introduction of these prominent resonances and have caused a shoulder in the total recombination rate.

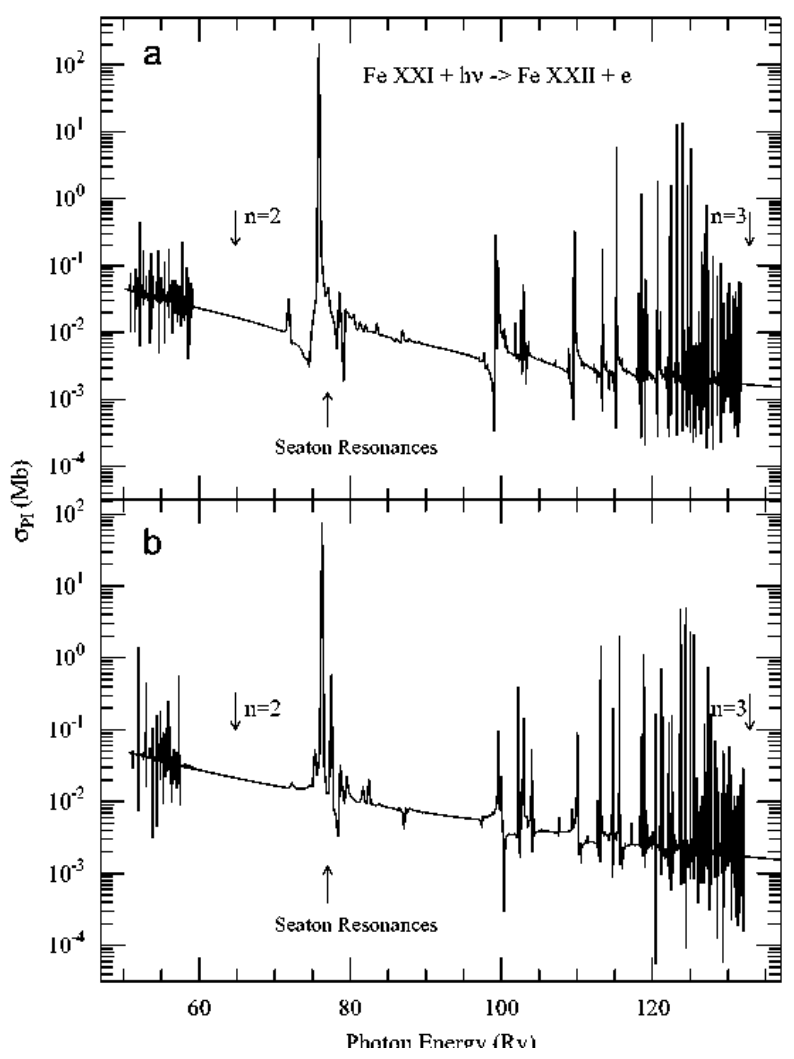

Fig. 2. Partial photoionization cross sections, $\sigma_{\mathrm{PI}}$, of the excited states of Fe XXI: (a) $2 s^{2} 2 p 3 d\left({ }^{3} D^{0}\right.$ ), (b) $2 s^{2} 2 p 3 d\left({ }^{3} F^{0}\right)$. The prominent Seaton or PEC (named by Seaton) resonances in the photon energy range between 73 and 82 Ry are due to various core transitions, e.g., $2 s^{2} 3 s\left({ }^{2} S\right) \rightarrow 2 s^{2} 2 p\left({ }^{2} P^{0}\right), 2 s 2 p 3 p\left({ }^{2} S\right) \rightarrow 2 s^{2} 2 p\left({ }^{2} P^{0}\right)$.

The prominent resonances in the energy range from about 73 to $82 \mathrm{Ry}$ for each state in Fig. 2 are PEC (photoexcitation-of-core) or Seaton resonances. These resonances were interpreted in detail and named PEC resonances by Seaton [9,29]. They are formed when the core ion absorbs photons for dipole allowed excitations which eventually follow to ionization as the core drops down to the ground state. The outer electron remains temporarily attached to a highly excited level as a spectator during this process. The phenomenon is inverse to DR, and manifests in a resonance, often wider, at the excited core threshold. Table 2 of core states indicates that there are eight possible PEC resonances in the energy range from about 73 Ry for $2 s^{2} 3 s\left({ }^{2} S\right) \rightarrow 2 s^{2} 2 p\left({ }^{2} P^{0}\right)$ transition to about 82 Ry for $2 s 2 p 3 p\left({ }^{2} S\right) \rightarrow 2 s^{2} 2 p\left({ }^{2} P^{0}\right)$ transition. These resonances contribute considerably to recombination rate and introduce a bump in the total 
recombination rate, as noted in the next section. Rest of the resonances are due to Rydberg series of autoionizing states belonging to $n=3$ core states.

\subsection{Total and state-specific recombination rate coefficients}

Both total and state-specific recombination rate coefficients of Fe XXI are reported. The state-specific recombination rates $\left(\alpha_{\mathrm{R}}(n L S)\right)$ for 685 bound states with $n \leq 10$ and $0 \leq 1 \leq 9$ are obtained for the first time. $\alpha_{R}(n L S)$ are needed for determination of level populations and formation of cascade matrices.

The values of individual state recombination rates at various temperatures depend on resonant and background features in the photoionization cross sections. Illustrative examples of $\alpha_{\mathrm{R}}(n L S)$ are given in Fig. 3 for states (a) $2 s^{2} 2 p^{2}\left({ }^{3} P\right)$, (b) $2 s 2 p^{3}\left({ }^{3} D^{0}\right)$, (c) $2 s^{2} 2 p 3 d\left({ }^{3} F^{0}\right)$, (d) $2 p^{4}\left({ }^{1} D\right)$, and (e) $2 s 2 p^{3}\left({ }^{5} S^{0}\right)$. Except for the ground state (Fig. 3a), all excited states exhibit explicit DR bumps generated by the resonant features in $\sigma_{\text {Рі }}(n L S)$. The equivalent electron states, such as in Figs. 3(b) and (d), provide dominant contributors to total recombination rate below $10^{6} \mathrm{~T}$ because of their low energy resonances in $\sigma_{\mathrm{PI}}$. The valence electron states, such as $2 s^{2} 2 p 3 d\left({ }^{3} F^{0}\right)$ in Fig. 3c, contribute depending on the strength of the resonances and enhanced background. In the example, $2 s^{2} 2 p 3 d\left({ }^{3} F^{0}\right)$ dominates in high temperature via dielectronic recombination because of the strong $n=3$ resonances in the high-energy region. The DR bump in the low temperature for ${ }^{5} S^{0}$. state is formed by the high-peak autoionizing resonances due to fine structure couplings (explained in the next section).

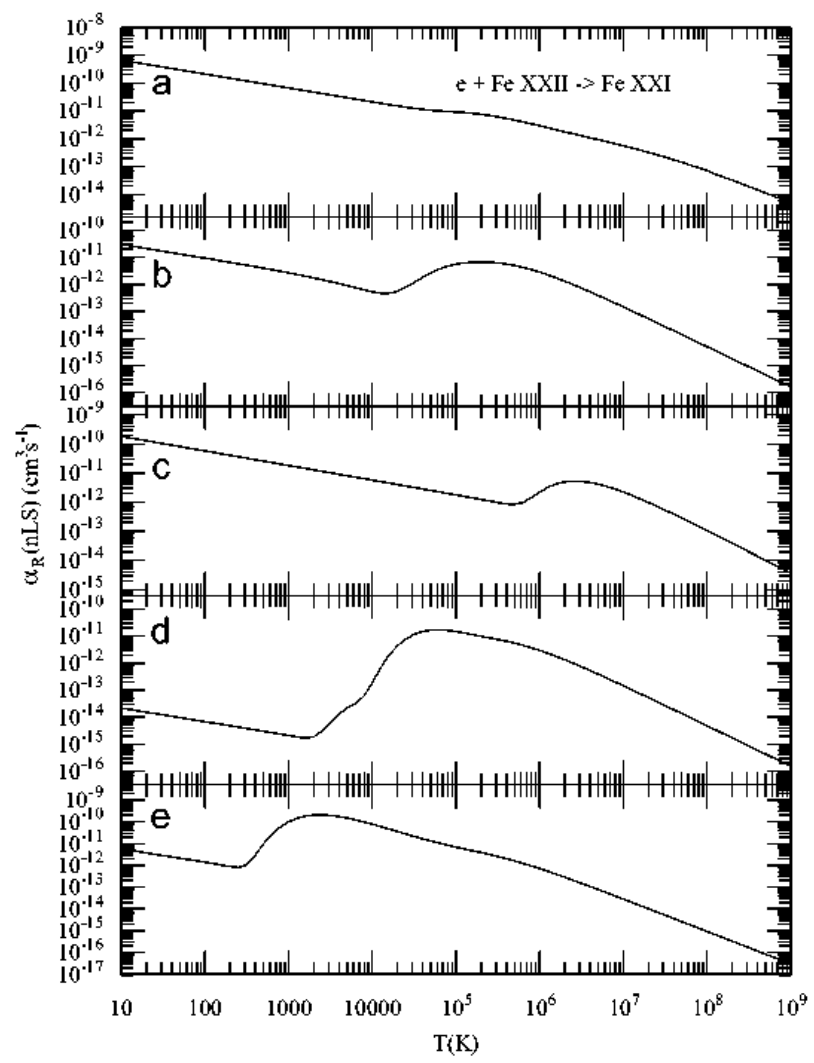

Fig. 3. State-specific recombination rate coefficients $\alpha_{\mathrm{R}}(n L S)$, including contributions of both RR and DR, of states (a) $2 s^{2} 2 p^{2}\left({ }^{3} P\right)$ : ground, (b) $2 s 2 p^{3}\left({ }^{3} D^{0}\right)$, (c) $2 s^{2} 2 p 3 d\left({ }^{3} F^{0}\right)$, (d) $2 p^{4}\left({ }^{1} D\right)$, and (e) $2 s 2 p^{3}\left({ }^{5} S^{0}\right)$. 
Table 3

Total recombination rate coefficients, $\alpha_{R}$ for $(e+$ Fe XXII $\rightarrow$ Fe XXI)

\begin{tabular}{|c|c|}
\hline $\log T(K)$ & $x_{R}\left(\mathrm{~cm}^{3} \mathrm{~s}^{-1}\right)$ \\
\hline 6.0 & $7.86 \mathrm{E}-11$ \\
\hline 6.1 & $7.04 \mathrm{E}-11$ \\
\hline 6.2 & $6.41 \mathrm{E}-11$ \\
\hline 6.3 & $5.97 \mathrm{a}-11$ \\
\hline 6.4 & $5.70 \mathrm{E}-11$ \\
\hline 6.5 & $5.52 \mathrm{E}-11$ \\
\hline 6.6 & $5.35 \mathrm{E}-11$ \\
\hline 6.7 & $5.10 \mathrm{E}-11$ \\
\hline 6.8 & $4.70 \mathrm{E}-11$ \\
\hline 6.9 & 4.20E 11 \\
\hline 7.0 & $3.62 \mathrm{~F}-11$ \\
\hline $\begin{array}{l}7.1 \\
7.2\end{array}$ & $3.02 \mathrm{E}-11$ \\
\hline 7.2 & $\begin{array}{l}2.45-11 \\
1.94 \mathrm{E}-11\end{array}$ \\
\hline 7.4 & $1.51 \mathrm{E}-11$ \\
\hline 7.5 & $1.15 \mathrm{E}-11$ \\
\hline 7.6 & $8.72 \mathrm{E}-12$ \\
\hline 7.7 & $6.53 \mathrm{E}-12$ \\
\hline 7.8 & $4.84 \mathrm{E}-12$ \\
\hline 7.9 & $3.57 \mathrm{E}-12$ \\
\hline 8.0 & $2.63 \mathrm{E}-12$ \\
\hline $\begin{array}{l}8.1 \\
8.2\end{array}$ & $1.91 \mathrm{E}-12$ \\
\hline 8.2 & $1.40 \mathrm{E}-12$ \\
\hline $\begin{array}{l}8.3 \\
8.4\end{array}$ & $\begin{array}{ll}1.02 \mathrm{~F} & 12\end{array}$ \\
\hline $\begin{array}{l}8.4 \\
8.5\end{array}$ & $\begin{array}{ll}7.43 \mathrm{~F} & 13 \\
5\end{array}$ \\
\hline $\begin{array}{l}8.5 \\
8.6\end{array}$ & $\begin{array}{l}5.40 \mathrm{E}-13 \\
3.93 \mathrm{E}-13\end{array}$ \\
\hline 8.7 & $2.86 \mathrm{E}-13$ \\
\hline 8.8 & $2.08 \mathrm{E}-13$ \\
\hline 8.9 & $1.52 \mathrm{E}-13$ \\
\hline 9.0 & $1.10 \mathrm{E}-13$ \\
\hline
\end{tabular}

The present state-specific rates should be accurate for most of the temperature range except at lower temperature where uncertainty may be introduced if resonances are not highly resolved and by slight shift of resonance positions. At higher temperature detailed features are damped out by the exponential factor in the $\alpha_{R}(n L S)$ integral. Based on the accuracy of the $R$ matrix method, energy comparison, and inclusion of interference effects, these rates are expected to be accurate within $50-70 \%$ above ${ }^{4} \mathrm{P}$ threshold, that is, above $6.7 \times 10^{5} \mathrm{~K}$, especially for dominant contributing states.

Values at various temperatures of the present unified total $\alpha_{R}(T)$, which include contributions of both the RR and DR, are presented in Table 3. The features are illustrated in Fig. 4 (solid curve) along with comparison with rates obtained by others. The top panel (a) of Fig. 4 presents $\alpha_{R}(T)$ overthe temperature range $10-10^{9}$ K while the bottom panel (b) details the hightemperature behavior, especially around the temperature $T_{M}$ of the ion's coronal abundance. The present $\alpha_{R}(T)$ (solid curve) is showing a characteristic rapid rise with decreasing temperature caused by very low energy recombination to the infinite number of high-n states, mainly due to RR. However, the DR rate coefficient $\alpha_{D R}(T)$ (long dashed curve) provided by Savin et al. [16], who integrated the measured low energy recombination spectrum, show very sharp rise with lower temperature and a broad structure. The present total $\alpha_{R}(T)$ is much lower than those by Savin et al. The largest difference is at about $2500 \mathrm{~K}$ where the recombination rate by Savin et al. peaks to $9.3 \times 10^{9}$. while the present rate is $1.1 \times 10^{9} \mathrm{~cm}^{3} / \mathrm{s}$. This could have resulted by some very sharp peak resonances in the near-threshold region seen in the experiment. Due to this discrepancy, Table 3 presents only high-temperature rates.

However, unified total $\alpha_{\mathrm{RC}}$ should be more accurate in the high-temperature region, 
especially around the temperature of abundance $T_{M} \sim 10^{7} \mathrm{~K}$, as it includes contributions of both the RR and DR and their interference effects in an ab initio manner. The DR rate by Savin et al. [16] merges with the present results at about $10^{6} \mathrm{~K}$ and decays quickly since the experiment was confined to low energy region only. DR rates using IRA by Gu [15] show almost complete agreement with the sharp rise in the low temperature rate obtained by Savin et al. The RR rate coefficient by Arnaud and Raymond [3] (dot-dashed) is considerably lower since it does not include any autoionizing resonances. However, at lower temperature it tends to merge with the present rates. The RR rates by $\mathrm{Gu}$ [10] and Badnell [11], which are basically equal to each other, are higher than those by Arnaud and Raymond at high temperature, but merge with the present rates at low temperature. The calculated DR rates from isolated resonance approach by Jacobs et al. [12] (dotted) and by Altun et al. [14] (short dash), which are similar to the earlier DR rates by Badnell [13], high-temperature rates by Gu [15] are in better agreement with the unified rates, but are lower. The enhancement in the recombination rate by $60 \%$ around the temperature of abundance is mainly due to the $n=3$ resonances in photoionization cross sections and may affect considerably the ionization balance of Fe XXI in plasmas in coronal equilibrium. As discussed in Nahar [17], present calculations included all channels with 28

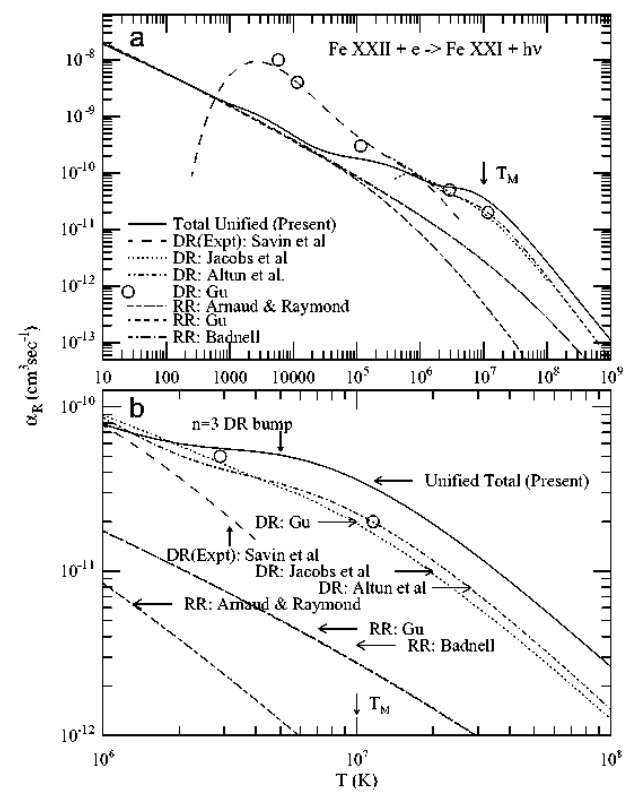

Fig. 4. Recombination rate coefficients of (e + Fe XXII $\rightarrow$ Fe XXI), (a) in low and high-temperature region, (b) in the high-temperature region. $T_{M}$ is the temperature of abundance in coronal equilibrium. Solid curve is unified total aR(T). DR rates: long dashed-from experiment by Savin et al. [16], open circle-by Gu [15], dot-by Jacobs et al. [12], dot and short dash-by Altun et al. [14]. RR rates: short and long dash-by Arnaud and Raymond [3], short dash-by Gu [10], dot and long dash-by Badnell [11].

core excitations (Table 1 ) and outer electron with $l \leq 9$. Photoionization includes autoionization to all levels via couplings of channels. Since all excited states are included for recombination, unified method essentially includes autoionization branching to ground as well as excited states. They were added by $n>10$ contributions resulting in the final DR peaks. Hence present calculation is complete with inclusion of all contributing channels to give rise the enhancement by a factor of 1.6 .

Total recombination rate coefficient with photoelectron energy, $\alpha_{\mathrm{R}}(E)$, of (e + Fe XXII 
$\rightarrow$ Fe XXI) can reveal detailed resonant structures in recombination process and the resonant part can be measured in storage rings. Fig. 5 presents unified $\alpha_{R}(E)$ going up to $2 s 2 p^{2}\left({ }^{2} D\right)$ threshold of Fe XXII. Panel (a) presents the contribution of $2 s 2 p^{3}\left({ }^{5} S^{0}\right)$ state alone (discussed in next section), panel (b) presents summed contributions of all triplets and singlets $\left({ }^{3,1} L\right)$ states of Fe XXI, and panel (c) is the total sum of (a) and (b). The arrow in Fig. 5c points the converging threshold $2 s 2 p^{2}\left({ }^{2} D\right)$ where DR peaks and then drops almost to zero due to core excitation. The figure shows considerable contributions by ${ }^{5} S^{0}$. state at very low energy compared to summed contributions of all triplets and singlets.

\subsection{Relativistic effects in low energy region}

Fe XXI can exist in low temperature photoionized plasmas. The low energy experiment for recombination of Fe XXI showed narrow and high-peak resonances near the ionization threshold [16] produced mainly by fine structure couplings of states that not allowed in LS coupling. In order to study these near-threshold resonant features in recombination rates, relativistic BPRM method with radiation damping [30] is employed to calculate the partial photoionization cross sections of $2 s^{2} 2 p^{3}\left({ }^{5} S^{0}\right)$ state of Fe XXI. In LS coupling, ${ }^{5} S^{0}$ does not couple to core ground $2 s^{2} 2 p\left({ }^{2} P^{0}\right)$ state of Fe XXII and hence cannot photoionize into it. However, in the low energy photoionization spectrum of carbon like O III, prominent narrow resonances were observed by Champeaux et al. [31] and was analyzed to arise through fine structure coupling of metastable $2 s 2 p^{3}\left({ }^{5} S^{0}\right)$ state [32]. Ionization potential of ${ }^{5} S^{0}$. is close to that of the ground state $2 s^{2} 2 p^{2}\left({ }^{3} P\right)$, and hence can be ionized by photons of comparable energy.

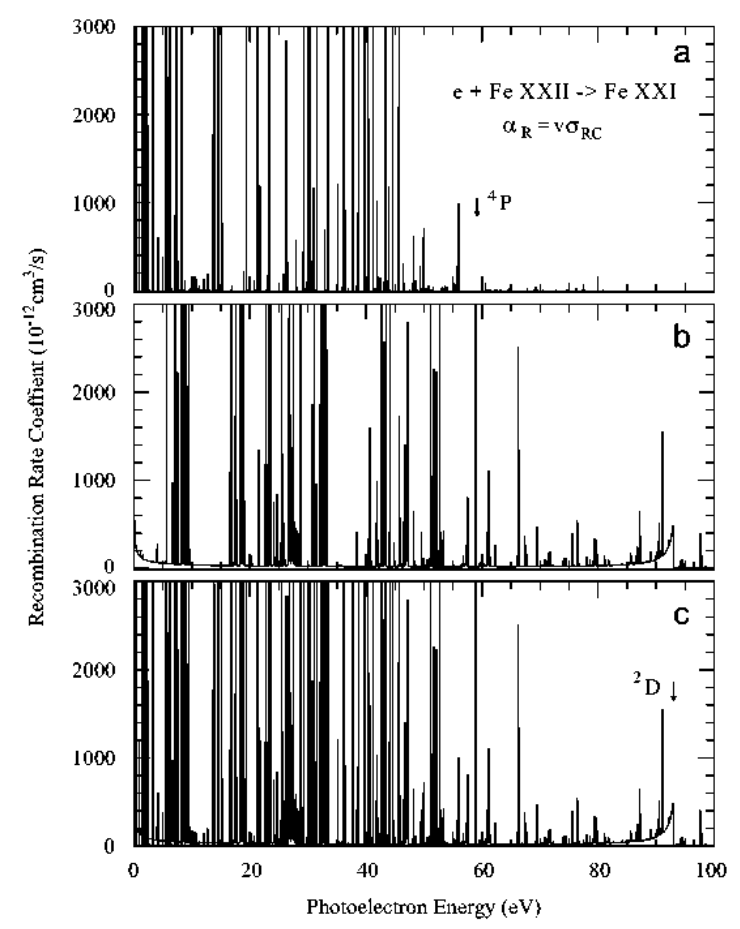

Fig. 5. Unified recombination rate coefficients $\alpha_{\mathrm{R}}$ of (e + Fe XXII $\rightarrow$ Fe XXI) with photoelectron energy. (a) Presents contributions of $2 s 2 p^{3}\left({ }^{5} S^{0}\right)$ state alone where extensive resonances below Fe XXII threshold $2 s 2 p^{2}\left({ }^{4} P\right)$ (pointed by an arrow) are produced from fine structure couplings. (b) Presents summed contributions of triplets and singlets $\left({ }^{3,1} L\right)$ states. (c) Is the total sum of (a) and (b). Arrow in (c) points converging threshold $2 s 2 p^{2}\left({ }^{2} D\right)$ where DR peaks and then drops due to core excitation. 
The partial photoionization cross sections of ${ }^{5} S^{0}$ are shown in Fig. 6. $\sigma_{\text {PI }}$ below the $2 s 2 p^{2}\left({ }^{4} P\right)$ core threshold (energy position is pointed by an arrow) is due to fine structure couplings only, that is, LS coupling will give no cross sections in this energy region. The $2 p$ electron of $2 s 2 p^{3}\left({ }^{5} S^{0}\right)$ state can photoionize only to $2 s 2 p^{2}\left({ }^{4} P\right) n d, n s\left({ }^{5} P\right.$ ) states by leaving the core at $2 s 2 p^{2}\left({ }^{4} P\right)$ state. However, through fine structure coupling $2 s 2 p^{2}\left({ }^{4} P\right) n d$, $n s\left({ }^{5} P_{3,2,1}\right)$ levels can couple to the J-levels of ${ }^{2} P^{0}{ }_{1 / 2,3 / 2} n p\left({ }^{1,3} P_{3,2,1}\right)$ and hence is allowed to radiationless transitions or autoionization. These relativistic mixings of channels generate below the threshold ${ }^{4} P$ many narrow but high-peak resonances with almost zero background cross sections as seen Fig. 6 . These resonances correspond to the extensive resonant structures in recombination rates in Fig. 5a and provide significant contributions to recombination rates at low temperatures. Above ${ }^{4} P$ threshold, the contribution of the state to recombination is almost zero.

Fig. 5c shows the detailed resonances in the recombination spectrum arising from recombination in to ${ }^{5} S^{0}$. state. Due to existence of an energy bandwidth or variable bandwidths in experimental measurements, each individual narrow resonances may not be detected, except features convolved with the bandwidths. However, the positions of resonant features observed in the experimental recombination spectrum [16] agree with those in Fig. 5c although some resonances close to the ionization threshold appear stronger in the experiment than the present calculated ones and are believed to be reasons for the differences in the total recombination rates. Although ${ }^{5} S^{0}$ provided significant contributions in low temperature, this state alone was not sufficient to match the high-peak broadened structure of $\alpha_{R}(T)$ obtained by Savin et al. [16]. Such broaden structure has not been seen for any other ion studied theoretically by the author. Summation of fine structure contributions by other states, not allowed in LS coupling, is expected to raise the total $\alpha_{\mathrm{R}}$ at low temperature. Nonetheless, the high-temperature rates are not affected by the fine structure details as they get damped by the exponential factor in recombination rate integral. The accuracy of those rates can be inferred from the general agreement among all other calculations.

Detailed comparison of the present $\alpha_{\mathrm{R}}(E)$ with an experiment will require carrying out full scale relativistic calculations for the low energy narrow resonances and then convolution of the resonances with the experimental beam function. The

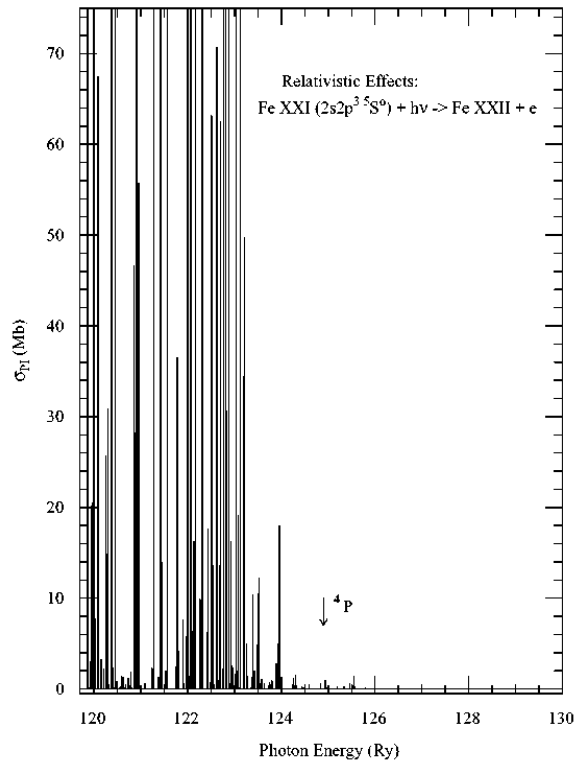

Fig. 6. Photoionization cross sections, $\sigma_{\mathrm{PI}}$, of the excited $2 s 2 p^{3}\left({ }^{5} S^{0}\right)$ state of Fe XXI. The narrow, high-peak resonances below the first excited core threshold $2 s 2 p^{2}\left({ }^{4} P\right)$ (pointed by arrow) are due to fine structure couplings. 
resonances due to fine structure couplings are very narrow compared to those in LS coupling. Considerable effort was needed with repeated calculations in various small energy bins, with very fine energy meshes ranging from $10^{-5}$ to $10^{-9}$. Ry, to resolve these narrow resonances of ${ }^{5} S^{0}$. state. Resonances become denser, narrower and more in number with fine structure. Each LS term of the core will have one or more number of fine structure components and each component will correspond to a Rydberg series making the computation a major task. It is in the future plan to carry out extensive relativistic BPRM calculations.

\section{Conclusion}

Electron-ion recombination and photoionization of (hv + Fe XXI $\leftrightarrow$ e + Fe XXII) are studied in detail with special emphasis on high-temperature recombination relevant to abundance of Fe XXI in most astrophysical sources. Unified total recombination rate coefficients $\alpha_{\mathrm{R}}(T)$, which include both RR and DR and the interference between them, are presented for the first time for Fe'XXI. State-specific recombination rate coefficients are presented for 685 bound states with $n \leq 10$ and $l \leq 9$ and are expected to be accurate within $50-70 \%$ for dominant states except probably at low temperature.

Photoionization cross sections with PEC resonances due to $n=3$ core thresholds in the energy region 73-82 Ry have introduced an enhanced shoulder in the recombination rates $\alpha_{R}(T)$. The enhancement is over 1.6 times of earlier rates at all temperatures $T>10^{6}$ K. This will have a bearing on the current two different interpretations of solar opacities.

The importance of fine structure couplings at very low energies is demonstrated through

$2 s 2 p^{3}\left({ }^{5} S^{0}\right)$ state. Although present recombination rate coefficient with photoelectron energy, $\alpha_{\mathrm{R}}(E)$, agrees well with the experiment, the differences in peaks of some resonances in low energy region have introduced large difference in the integrated rate coefficients in low temperature region. Contributions from fine structure couplings from other levels are expected to raise the low temperature total rates.

The present results can be improved in two ways: (i) using relativistic fine structure couplings, especially in the low temperature region, and (ii) resolving resonances with finer energy mesh. Neither of these should have a significant effect on present results at high temperature. Inclusion of relativistic fine structure will double the number of 835 bound states to 1611 bound fine structure levels [33]. In addition, fine structure will increase the number of core excitations leading to increment of Rydberg series of autoionizing resonances and hence will require finer resolution. These improvement will require extensive computations and is a part of the future work.

Photoionization and recombination data will be available electronically at NORAD website: www.astronomy. ohio-state.edu/nahar/nahar_radiativeatomicdata/index.html.

\section{Acknowledgments}

This work was partially supported by the NASA Astronomy and Physics Research Analysis Program. The computational work was carried out on Cray machines at the Ohio Supercomputer Center in Columbus Ohio. 


\section{References}

[1] Pallavicini R, Serio S, Vaiana GS. A survey of soft X-ray limb flare images-the relation between their structure in the corona and other physical parameters. Astrophys $\mathrm{J}$ 1977;216:108-22.

[2] Guainazz M, Bianch S. On the origin of soft X-rays in obscured AGN: answers from high-resolution spectroscopy with XMM-Newton. Mon Not R Astron Sci 2007;374(4):1290-302.

[3] Arnaud M, Raymond J. Iron ionization and recombination rates and ionization equilibrium. Astrophys J 1992;398:394-406.

[4] Dopita MA, Sutherland RS. Astrophysics of the diffuse universe. Berlin: Springer; 2003.

[5] Nahar SN, Pradhan AK. Electron-ion recombination rate coefficients, photoionization cross sections, and ionization fractions for astrophysically abundant elements I. Carbon and nitrogen. Astrophys J Suppl 1997;111:339-55.

[6] Nahar SN, Pradhan AK. Electron-ion recombination in the close-coupling approximation. Phys Rev Lett 1992;68:1488-91.

[7] Nahar SN, Pradhan AK. Unified treatment of electron-ion recombination in the closecoupling approximation. Phys Rev A 1994;49:1816-35.

[8] Nahar SN, Pradhan AK. Unified electron-ion recombination rate coefficients of silicon and sulfur ions. Astrophys J 1995;447:966-79.

[9] Nahar SN, Pradhan AK. Radiat Phys Chem 2004;70:323.

[10] Gu MF. Radiative recombination rate coefficients for base through F-like isosequences of Mg, Si, S, Ar, Ca, Fe, Ni. Astrophys J 2003;589:1085-8.

[11] Badnell N. Radiative recombination data for modeling dynamic finite-density plasmas. Astrophys J Suppl 2006;167:334-42.

[12] Jacobs VL, Davis J, Kepple PC, Blaha M. The influence of autoionization accompanied by excitation on dielectronic recombination and ionization equilibrium. Astrophys $\mathrm{J}$ 1977;211:605-16.

[13] Badnell N. Dielectronic recombination of Fe22+ and Fe21+. J Phys B 1986;19:3827-35.

[14] Altun Z, Yumak A, Badnell NR, Colgan J, Pindzola MS. Dielectronic recombination data for dynamic finite-density plasmas-VI. The boron isoelectronic sequence. Astron Astrophys 2004;420:775-81.

[15] Gu MF. Dielectronic recombination rate coefficients for H-like through Ne-like isosequences of Mg,Si, S, Ar, Ca, Fe, Ni. Astrophys J 2003;590:1131-40.

[16] Savin DW, Kahn SM, Gwinner G, Grieser M, Repnow R, Saathoff G, et al. Dielectronic recombination of Fe xxi and Fe xxii via $N-2-N^{1}-2$ core excitations. Astrophys $\mathrm{J}$ Suppl 2003;147:421-35.

[17] Nahar SN. Photoionization cross sections of Fe XXI. JQSRT 2008;109:2417-26.

[18] Nahar SN. Electron-ion recombination rate coefficients for Si I, Si II, S II, S III, CII, and C-like ions C I, N II, O III, FIV, Ne V, Na VI, Mg VII, Al VIII, Si IX, and S XI. Astrophys J Suppl 1995;101:423-34.

[19] Nahar SN. Electron-ion recombination rate coefficients for Si I, Si II, S II, S III, C II, and C-like ions C I, N II, O III, F IV, Ne V, Na VI, Mg VII, Al VIII, Si IX, and S XI. Erratum Astrophys J Suppl 1996;106:213-4.

[20] Nahar SN. Electron-Ion recombination rate coefficients and photoionization cross sections for astrophysically abundant elements VIII. Ar XIII with new features. 
Astrophys J Suppl 2004;156:93-103.

[21] The Opacity Project Team. The opacity project, vol 1,1995, vol. 2,1996. Institute of Physics Publishing.

[22] Hummer DG, Berrington KA, Eissner W, Pradhan AK, Saraph HE, Jully JA. Atomic data from the IRON project. 1: goals and methods. Astron Astrophys 1993;279:298-309.

[23] Bell RH, Seaton MJ. Dielectronic recombination: I. General theory. J Phys B 1985;18:1589-629.

[24] Nahar SN. Total electron-ion recombination for Fe III. Phys Rev A 1996;53:2417-24.

[25] Eissner W, Jones M, Nussbaumer H. Techniques for the calculation of atomic structures and radiative data including relativistic corrections. Comput Phys Commun 1974;8:270306.

[27] NORAD website: (www.astronomy.ohiostate.edu/nahar/nahar_radiativeatomicdata/index.html).

[28] Luo D, Pradhan AK. Atomic data for opacity calculations. XI-the carbon isoelectronic sequence. J Phys B 1989;22:3377-95.

[29] Yu Y, Seaton MJ. Atomic data for opacity calculations. IV. Photoionisation cross sections for C II. J Phys B 1987;20:6409-29.

[30] Zhang HL, Nahar SN, Pradhan AK. Close coupling R-matrix calculations for electron-ion recombination cross sections. J Phys B 1999;32:1459-79.

[31] Champeaux J-P, BizauJ-M, Cubaynes D, Blancard C, Nahar SN, Hitz D, et al. Measurements and calculations of photoionization cross sections of multiply-charged ions in ground and metastable states along the isonuclear series of oxygen: $\mathrm{O}^{2+}$ to $\mathrm{O}^{4+}$. Astrophys J 2003;148:583-92.

[32] Nahar SN. Photoionization cross sections of O II, O III, O IV, and O V: benchmarking Rmatrix theory and experiments. Phys Rev A 2004;69:042714-1-9.

[33] Nahar SN. Atomic data from the Iron Project XLV. Relativistic transition probabilities for carbon-like Ar XIII and Fe XXI using Breit-Paili R-matrix method. Astron Astrophys Suppl Ser 2000;127:253-70. 